\title{
Effectiveness of Intra-dialytic Stretching Exercises on Leg Muscle Cramp among Hemodialysis Patients
}

\author{
Samah Saad Salem ${ }^{1,2}$, Shaimaa Mohamed Elhadary ${ }^{1}$ \\ ${ }^{1}$ Medical-Surgical Nursing Department, Faculty of Nursing, Cairo University, Egypt \\ ${ }^{2}$ Medical-Surgical Nursing, College of Nursing, King Saud bin Abdul-Aziz University for Health Sciences, \\ Riyadh, Saudi Arabia
}

\begin{abstract}
End stage renal disease (ESRD) is a chronic disease needing routine hemodialysis (HD) to substitute the failed kidneys' functions. Leg muscle cramps is commonly associated with HD. the aim of the study was to evaluate the effectiveness of intra-dialytic stretching exercises on leg muscle cramp among hemodialysis patients. A quasi experimental design one group pre-post was used to evaluate the effectiveness of intra-dialytic stretching exercises on leg muscle cramp among hemodialysis patients at Kasr El-Einy Center for Urology, Nephrology and Renal Transplantation. A convenience sample of 60 patients with ESRD and on regular HD was recruited in the study. Data were collected using data collection instrument that consisted of 2 parts: 1) Demographic and biomedical data sheet 2) Cramp assessment sheet. The study results revealed that there was a significant difference between the study participants leg cramps intensity and frequency before and after application of leg stretching exercises with $t=8.27$ at $p$ value $=0.000 \& t=5.22$ at $p$ value $=0.000$ respectively. In conclusion; Intra-dialytic stretching exercise is an effective intervention to reduce leg muscle cramp among hemodialysis patients. Further studies are recommended to explicate the effectiveness of intradialytic stretching exercises on leg muscle cramp among hemodialysis patients in different health care settings.
\end{abstract}

Keywords: hemodialysis, intra-dialytic, leg muscle cramp, stretching exercises

\section{Introduction}

End stage renal disease (ESRD) is a progressive, devastating, chronic disease needing nursing and medical interventions. The development of the disease influences quality of life, physical and mental wellbeing, functional status, level of independency and personal relations and social functioning [1].

Hemodialysis (HD) is a procedure used to manage patients with ESRD through using artificial kidney machine to substitute the failed kidneys' functions [2]. HD, is considered one of the principal renal replacement therapies among patients with ESRD, it is a time-consuming procedure that precedes around 3 to 5 hours a day, 2 or 3 times per week [3]. One of the most common complications amongst the HD patients is Muscle Cramps. It can happen anywhere in the body, but most common in calf muscles, feet, toes, thigh and abdomen respectively. Just about all HD patients complain of leg muscle cramps at certain time during HD sessions [4].

Muscle cramps, involuntary muscle contraction accompanying with severe stretched pain, most commonly associated with HD. Around $33 \%$ to $86 \%$ of patients receiving HD have experienced muscle cramps which begin with very painful muscle twitches causing the patient hardly to move. Unluckily, this can happen during HD treatment. Usually the muscle cramp can lead to termination of dialysis session before the planned time, causing a less effective treatment. There are many factors that may lead to muscle cramps in patients with ESRD receiving dialysis as a decreased fluid volume in the body, decreased blood pressure, fluid and electrolyte imbalance, decreased sodium level, tissue hypoxia and hypomagnesaemia [5].

Intra-dialytic muscle cramps during HD may also be due to too much dialysis ultrafiltration (UF), and raise of serum creatine kinase (CK) that may cause an abnormal use of energy by muscles and therefore leading in muscle cramps. Numerous treatment methods for HD related cramps have been suggested, however most of them have been accompanying with severe side effects and none have been unconvincingly effective. It concluded that, stretching exercises for the affected muscle can immediately relief the cramp. The usage of consistent stretching exercises to avoid occurrence of cramps was proposed and concluded that, muscle stretching during cramp could be helpful for HD patients [6].

Ever since cramps are a common complication for HD patients that cause discomfort. So proper interfering with muscle cramps and preventing the occurrence as well become an essential intervention of the nurses who are taking care of HD patients during that time almost everywhere [7]. Exercise is one of the probable preventive measures to decrease the loss of muscle protein and maintenance of muscle functions. In recent times, several studies have revealed the importance of exercise and physical activities to stop muscle wasting in ESRD patients. There are many exercise protocols using different exercise intensities, frequencies, and duration. Though, the exercise protocols generally consist of two to three times per week during HD, with moderate intensity lasting around 30 minutes, and for 8 weeks to 12 months [8]. 
There is some debate as to the risk-benefit ratio of the pharmacological treatment of leg cramps. These drug as quinine has considerable, potentially dangerous side effects. Careful monitoring for the effectiveness and toxicity are warranted. If the quinine is ineffective, or if there is any evidence for adverse effects, so it should be discontinued. Other Studies suggest that vitamin E may be a drug for the management of leg cramps for patients receiving dialysis. However, vitamin $\mathrm{E}$ interactions with anticoagulants as warfarin or heparin, and a concern for adverse effects for risk of bleeding may limit its use in dialysis patients [9].

Non-pharmacological strategies can be employed in order to prevent or reduce cramps in dialysis patients. On pharmacological strategies can be a local massage of the affected muscle and the application of moist heat may provide some comfort. Other low-risk strategies include performing stretching exercises, performing mild exercise such as riding a stationary bicycle during dialysis or prior to bedtime, minimizing alcohol and caffeine. Also keeping bed covers loose and not tucked in to prevent cramps. Local heat including showers or baths or ice, massage, walking or leg jiggling followed by leg elevation, are other methods reported to help relieve muscle cramps. Such strategies have a better patient outcome and fewer side effects [10]. It is reported that ESRD affects an increasing number of populations with the estimated incidence about $15 \%$ of adults in the United States. Stretching exercise or regular physical activities is highly required for HD patients with ESRD. Though, in spite of the many helpful effects of exercise in 64 intra-dialysis exercise programs provided for HD Patients, exercise programs are still not well practiced or even provided in the majority of hospitals [11].

The physical activity program for HD patients is aiming for conservation or improvement of physical fitness to achieve patient's independence, increase social integration, as well as improve the quality of life. Physical activity programs must include activities that leading to joint flexibility maintenance and muscular strength, avoid muscle dis-balances, improvement of muscle coordination, and cardio-respiratory functions. Other benefits of physical activity like improvement of mental status, level of independency, better dealing with chronic disease and the required treatment (decrease the length of hospitalization and morbidity and mortality). Achievement of physical activity programs goals is very crucial for most of HD population [12].

Physical activity programs could be divided into separate exercises according to the availability of time within the complex HD treatment. Each one of the activity program is distinctive due to diverse acceptance of the treatment complexity and the disease itself [13]. German expert in nephrology field divided physical activity programs into numerous groups consistent with the specific concern on individual components of physical fitness, training of balance-coordination, Breath exercises, relaxation techniques, isometric exercises during HD and stretching exercises. The goal of stretching exercises is to improve general muscle strength, enhance muscle volume, decrease muscle fibers' atrophy, support bone tissue' mineralization, and reduce the muscle cramps [14].

\section{I.1. Significance of the Study:}

During the last 2 decades, the annual growth in number of dialysis patients was $6-12 \%$ in many developed countries and the growth rate increase in several developing countries. Nearly 0.5 million patients are treated with dialysis [15]. Most Egyptian dialysis centers whether governmental or private are under supervision by the Egyptian Ministry of Health are equipped with dialysis machines but there is no Egyptian guidelines to standardize practices related to patient's problems during dialysis session which leads to low patient satisfaction and low quality of patient care[16]. Leg cramps begin with muscle twitches, and can be very painful in any circumstances. Leg cramps during hemodialysis are painful involuntary spasm that can produce patient discomfort which leads to premature termination of dialysis session earlier than planned, non-compliance with the prescription and therefore under dialysis. Thus interfering with the muscle cramps and even reducing or preventing the occurrence becomes a major responsibility of the patients. Since nurses are taking care of hemodialysis patients almost everywhere, it becomes predominantly the nurses' role. The finding of this study might be useful in using low cost, low side effect treatment for HD patients. It also may contribute to high patient's satisfaction. This will lead to improve quality of patient care.

\section{Material and Methods}

The current study aims to investigate the effectiveness of intra-dialytic stretching exercises on leg muscle cramp among hemodialysis patients.

The following research Hypotheses were postulated in relation to the aim of the study:

$\mathrm{H}_{1}$ : There will be a significant difference in the intensity of leg muscle cramps before and after application of intra-dialytic stretching exercise among Hemodialysis patients.

$\mathrm{H}_{2}$ : There will be a significant difference in the frequency of leg muscle cramps before and after application of intra-dialytic stretching exercise among Hemodialysis patients.

$\mathrm{H}_{3}$ : There will be a significant difference in the frequency of sleep disturbance during hemodialysis session before and after application of intra-dialytic stretching exercise among Hemodialysis patients. 


\subsection{Design}

One group quazi-Experimental pre-posttest design will be used to achieve the aim of the current study. This design is one of the experimental designs in which data are collected from research subjects both before and after introducing an intervention. In the current study, this design will help to determine the existing intensity of leg cramps before intervention and evaluate the effect that will occur after application of intradialytic stretching exercises on leg muscle cramp among hemodialysis patients [17].

\subsection{Setting}

The current study was conducted in Kasr El-Einy Center for Urology, Nephrology and Renal Transplantation which is affiliated to Cairo University, Egypt in which the hemodialysis provided for free for Egyptian patients with ESRD.

\subsection{Sample:}

A convenience sample of 60 patients with end stage renal disease (ESRD) and on regular HD and having developed leg cramp during the HD were recruited in the current study according to the following inclusion \& exclusion criteria.

\section{Inclusion criteria}

- Adult, with an aged range between 20 - 60 years

- Male or female

- Having leg cramps during hemodialysis

- Willing to participate in the study and how the ability to communicate.

Exclusion criteria

- Patients having any other chronic disease

- Having malignancy as hepatic or liver failure.

- Patient with border values of potassium (hypokalemia $<3.5$ or hyperkalemia $>6 \mathrm{mmol} / \mathrm{l}$ will be excluded from the study.

\subsection{Data collection tools:}

To achieve the aim of the study, data were collected using the following instruments;

1. Demographic and biomedical data sheet; developed by the researchers and include variable as: age, sex, marital status, occupation, BMI, smoking habits, number of dialysis sessions /week, duration of dialysis, ...etc.

2. Cramp assessment scale; developed by American Physical Therapy and updated at 2012. It includes variables as Level of muscle cramp, Frequency of the cramp, location, affection on sleep disturbance and patient evaluation of leg cramps. It is a self-report measure with items on 4-point scales developed to rate cramps intensity as: nil $=0$, mild $=1$, moderate $=2,3=$ severe.

3. Intra-dialysis stretching leg exercises for cramps. Adopted from research exercise therapy center, faculty of physical education. Charles University [18]. It includes nine different stretching exercises; 1. Ankles 2. Knees 3. Hip flexors 4. Inner thigh muscles 5. Outer thigh muscles 6. Front thigh muscles 7. Back of thigh muscles 8. Gluteal muscles \& 9. Deep back muscles. The goal of these leg stretching exercises is to improve overall muscle strength, increase muscle volume, reduce atrophy of muscle fibers and support mineralization of the bone tissue.

\subsection{Pilot study}

A pilot study was done with 6 patients to assess the instrument's feasibility and applicability and to test needed time for filling the tools. Required modifications were done accordingly and those patients were excluded from the study.

\subsection{Ethical considerations}

After taking the approvals from the authoritative person at Kasr El-Einy Center for Urology, Nephrology and Renal Transplantation to conduct the current study a written informed consent were obtained from each participant prior starting the data collection. Each participant was informed about the purpose of the study and participant's anonymity was kept as well as the confidentiality of the data. Each participant was informed that he has the right to withdraw from the research at any time or refuse to share without any affection in their treatment. 


\subsection{Procedure for data collection:}

The current study was conducted over three phases: a) preparation, b) implementation and c) evaluation. Preparation phase included obtaining an official permission from the head of Urology, Nephrology and Renal Transplantation Center. Also preparation of study tools \& stretching leg exercises .The adopted leg stretching exercises was consulted with the physician in charge to provide additional information regarding patients' health status. This information as ESRD courses, co-morbidities, pharmacotherapy, specific dialysis anamnesis (frequency and duration of dialysis session).

In implementation phase, the first tool related to demographic and biomedical data were completed for all participants. The investigator measured the baseline data related cramps using the second tool, cramps assessment scale. The investigator then performed stretching exercises to the study participants after the first hour of each dialysis session for 3 consequential three weeks. These exercises were performed on an individual base for each participant on a short session of about 20-30 minutes. At the end of the three weeks re-evaluation of leg gramps was done using cramps assessment scale to compare before and after intervention.

\subsection{Statistical analysis:}

Data was entered and analyzed using Statistical Package for Social Science software (SPSS version 22); Data related descriptive statistics were summarized using mean as an average, standard deviation as a measure of dispersion of result around the mean. Also frequency and percentage of for each variable studied. T test was used for comparison of mean. The alpha level of .05 was utilized for all tests of significance. The internal consistency of all tools was conducted by Cronbach alpha.

\section{Results}

Section I: Demographic and medical related data.

Figure (1): Frequency \& percentage distribution of gender among studied participants $(\mathrm{n}=60)$

\section{Gender}

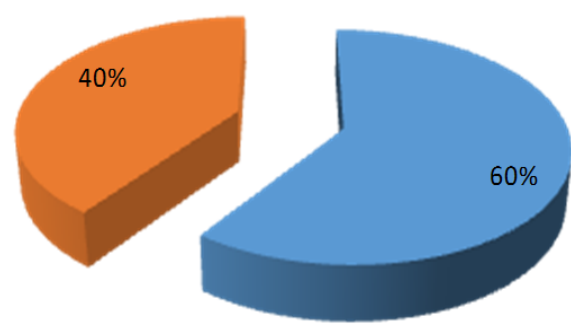

male

n female

Table (1): Frequency \& percentage distribution of medical data among studied participants ( $\mathrm{n}=60$ )

\begin{tabular}{|c|c|c|}
\hline Variables & No. & $\%$ \\
\hline \multicolumn{3}{|l|}{ Gender } \\
\hline Male & 40 & 60 \\
\hline Female & 20 & 40 \\
\hline \multicolumn{3}{|l|}{ Age } \\
\hline $20->40$ & 20 & 33.3 \\
\hline $40-60$ & 40 & 66.7 \\
\hline $\mathrm{M} \pm \mathrm{SD}$ & $45.38 \pm 9.61$ & \\
\hline \multicolumn{3}{|c|}{ Duration of kidney problem. } \\
\hline$<1$ year - & 2 & 3.3 \\
\hline 3 years - & 8 & 13.3 \\
\hline 6 year & 42 & 70 \\
\hline 9 years -12 year & 8 & 13.3 \\
\hline $\mathrm{M} \pm \mathrm{SD}$ & $6.21 \pm 2.27$ & \\
\hline \multicolumn{3}{|c|}{ Duration of hemodialysis session. } \\
\hline 3 hours & 10 & 16.7 \\
\hline 4 hours & 50 & 83.3 \\
\hline \multicolumn{3}{|c|}{ Frequency of hemodialysis session per week. } \\
\hline 3 times per week & 59 & 96.7 \\
\hline 4 times per week & 1 & 3.3 \\
\hline
\end{tabular}

Section II: Hypothesis testing and other supportive findings. 
Table (2): Comparison of intensity of leg cramps scores among studied participants before and after leg stretching exercises $(\mathrm{n}=60)$

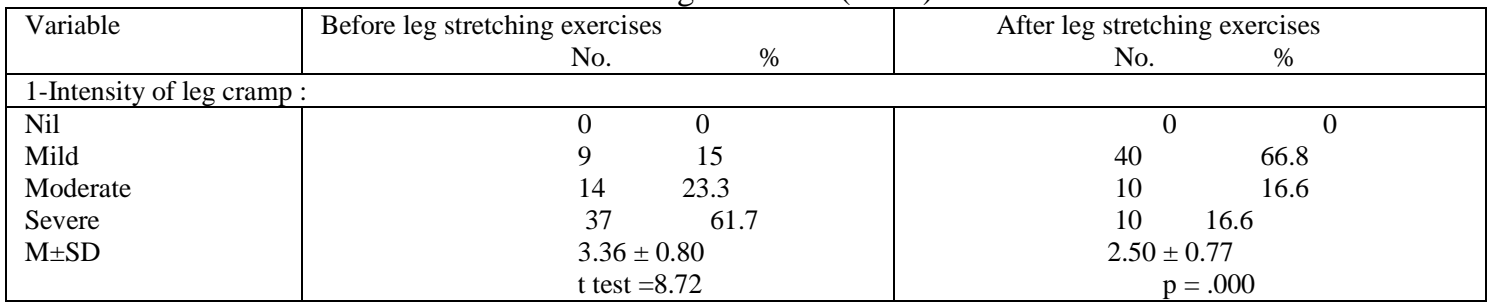

Table (3): Comparison of frequency of leg cramps among studied participants before and after leg stretching exercises $(n=60)$

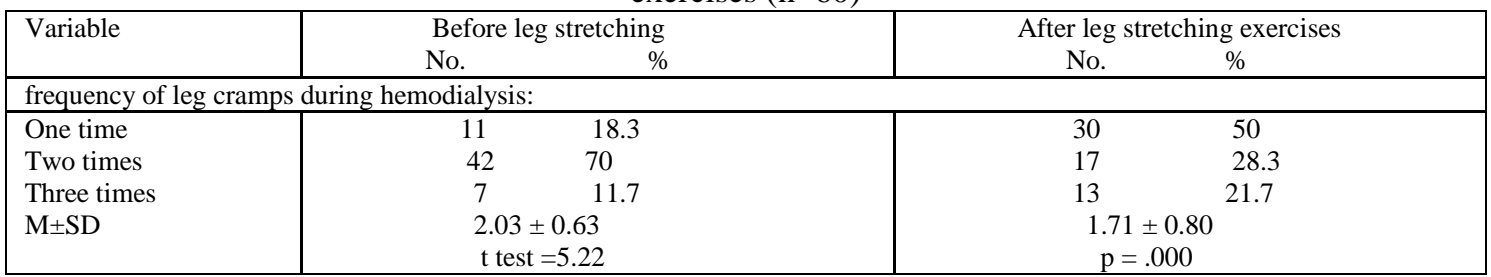

Table (4): Comparison of frequency of sleep disturbance scores during hemodialysis session among studied participants before and after leg stretching exercises $(n=60)$

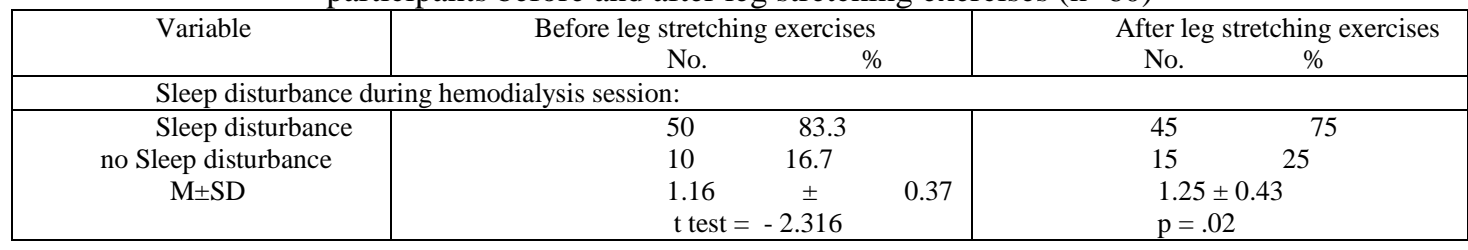

Table (5): Participants' evaluation of leg cramps developed after leg stretching exercises ( $\mathrm{n}=60)$

\begin{tabular}{|l|c|c|}
\hline & No. & $\%$ \\
\hline Variable & \multicolumn{2}{|c|}{} \\
\hline Participants' evaluation of leg cramps & 10 & 11.6 \\
Completely free of symptoms & 40 & 66.7 \\
Improved & 3 & 5 \\
Unchanged & 7 & 11.6 \\
Worsened & & \\
\hline
\end{tabular}

Table (1) illustrated that $(60 \%)$ of the study participants were male whereas $(40 \%)$ were female. (33.3\%) of the studied participants were 20 - > 40 years whereas $(66.7 \%)$ of them were from $40-60$ years old with an $\mathrm{M}+\mathrm{SD}=45.38+9.61$. It was found that $(70 \%)$ of the study participants were had kidney problems since 6 years - less than 9 years. Also (83.3\%) of the study participants had a 4 hours dialysis session. $(96.7 \%)$ of the study participants had a 3 times dialysis session per week.

Table (2) revealed that $(61.7 \%)$ of the study participants had severe intensity of leg cramps before intervention. While $(66.8 \%)$ of the study participants had mild intensity of leg cramps after application of leg stretching exercises, with a significant statistical differences $(\mathrm{t}=8.23$ at $\mathrm{p}=0.000)$.

As regards to frequency of leg cramps, table (3) showed that (70\%) of the study participants had leg cramps two times before intervention and $(50 \%)$ of the study participants had leg cramps one time after application of leg stretching exercises. There was significant statistical differences as regards leg cramps before and after application of leg stretching exercises, with a $(t=8.23$ at $\mathrm{p}=0.000)$.

As regards to sleep disturbance during hemodialysis session, table (4) revealed that $(83.3 \%)$ of the study participants had sleep disturbance before intervention and $(75 \%)$ of the study participants had sleep disturbance after application of leg stretching exercises. There was no significant statistical differences before and after application of leg stretching exercises, with $\mathrm{a}(\mathrm{t}=-2.31$ at $\mathrm{p}=0.02)$.

Regarding participants' evaluation of their leg cramps after application of stretching leg exercises, table (5) revealed that $(66.7 \%)$ of the study participants reported an improvement while $(11.3 \%)$ of the study participants reported a worsened leg cramps after the intervention. 


\section{Discussion}

The current study revealed that more than half of patients were from 40 - 60 years old these results was matched with study in USA done to evaluate the Intra-dialytic Massage for Leg Cramps Among Hemodialysis Patients: a Pilot Randomized Controlled Trial in (2016) and revealed that the mean age of the participants exceeded the 50 years [19].

Results of this study supported the hypothesis of significant difference in the intensity of leg muscle cramps before and after application of intra-dialytic stretching exercise among hemodialysis patients. These findings were supported by another study finding which conducted to evaluate the effectiveness of intra-dialytic stretching exercise on reducing muscle cramps among hemodialysis patients at sri manakula vinayagar medical college and hospital, Puducherry and reported that after the administration of Intra-dialytic Stretching Exercise the level of muscle cramps was reduced to 05.44 with the standard deviation of 2.96 [20].

It was conducted a study on Hemodialysis related leg cramps are common among older patients and the best treatment includes stretch exercises from the East Carolina, United States of America. The study results showed that $85 \%$ of patients' symptoms was relieved effectively by practicing the stretching exercises during the Hemodialysis [21]. Another study conducted on intra-dialytic exercise programs on the Hemodialysis unit across the Canada country. Around 3500 Hemodialysis centers consists of 1,02,567 patients were participated in this exercise programs during the course of study. However this exercise shows the positive outcomes towards on reducing the muscle cramps during the dialysis programs [22].

Regarding the frequency of leg cramps, the current study showed that the majority of the study participants had leg cramps two times before intervention while after of participants application of leg stretching exercises half of them had leg cramps one time with significant statistical differences between before and after application of exercises, The study findings are consistent with the study results done by [23] who conducted a study to evaluate the effectiveness of intra-dialytic massaging on leg cramping among hemodialysis patients with total number of $32 \mathrm{HD}$ patients with recurrent leg cramps during treatment. The study results presented that the patients in the study group reported decreased incidence of leg cramping during dialysis than the control group. This study discovered that the intra-dialytic massaging was effective to reduce muscle cramps during dialysis.

As regards to sleep disturbance during hemodialysis session, the study results revealed that the majority of the study participants had sleep disturbance before and after the intervention in congruent with the study done to assess the impact of stretching exercises protocol on reduction of muscle cramping during hemodialysis, among chronic renal failure patients and found that that the majority of patient $66.67 \%$ answered the cramp leads to sleep disturbance [24].

Regarding participants' evaluation of their leg cramps after application of stretching leg exercises, more than half of the study participants reported an improvement after the intervention. In the same line a study conducted at 2016 which conducted to assess the effectiveness of intra-dialytic stretching exercise on muscle cramps among patients undergoing hemodialysis who reported that, the leg stretching exercise was effective on reduction of muscle cramps among patients enduring hemodialysis and revealed that the average paired $t$ test assessment of interventional group was 17.25, during 1st to 5th days of hemodialysis session, which be there highly significant at $\mathrm{p}<0.05$ level [25].

These findings were also supported by another study which conducted at 2012 to assess the effectiveness of intra-dialytic stretching exercise on muscle cramps among patients undergoing hemodialysis in a selected hospital at Mangalore. This study used a quasi-experimental design with purposive sample of 30 patients with ESRD. The result reported that there was a significant difference for muscle cramps score in pre and posttest for the interventional group $(50.297, \mathrm{p}<0.05 \%)$. This study revealed that intra-dialytic stretching exercise was effective technique in reduction of muscle cramps among ESRD patients undergoing hemodialysis [26].

\section{Conclusion}

This study implies that most of ESRD patients undergoing hemodialysis had frequent leg muscle cramps with variant intensity during the HD session. After applying stretching exercise session for those patients, it revealed a significant level of reducing of muscle cramps was found. Thus it is concluded that, intradialytic stretching exercise is an effective technique and simple and safe strategy in reduction of muscle cramps among patients undergoing hemodialysis.

\section{Recommendations}

1. Further studies are required to explicate the effectiveness of intra-dialytic stretching exercises on leg muscle cramp among hemodialysis patients in different health care settings.

2. The same study can be conducted using patient effective experimental design.

3. The study can be replicated including larger sample size for better generalization. 
4. The study can be conducted for peritoneal dialysis patients.

5. Nurses should learn how to implement the leg stretching exercises to be applied for hemodialysis patients.

\section{Acknowledgment}

Grateful thanks for patients how accepted to participate in the current study. The researchers also appreciated the study's reviewers for their constructive remarks which really made the article stronger.

\section{References}

[1]. Cleary J1, Drennan J., (2005). Quality of life of patients on haemodialysis for end-stage renal disease. Journal of Advanced Nursing. Sep;51(6):577- 86

[2]. Caplin B, Kumar S, Davenport A. (2011) .Patients' perspective of haemodialysis-associated symptoms. Nephrol Dial Transplant ;26(8):2656-63.

[3]. Park, S. \& Jung, T, (2011). Intradialytic Exercise Programs for Hemodialysis Patients. Chonnam Medical Journal ;47:61-65

[4]. Danasu, R. (2016). Effectiveness of intra-dialytic stretching exercise on reducing muscle cramps among hemodialysis patients at sri manakula vinayagar medical college and hospital, puducherry. International Journal of Information Research and Review Vol. 03, Issue, 06, pp. 2443-2445.

[5]. Damasiewicz MJ, Polkinghorne KR.(2011). Intra-dialytic hypotension and blood volume and blood temperature monitoring. Nephrology (Carlton) 2011;16:13-8.

[6]. Ulu, M. \& Ahsen, A, (2015). Muscle Cramps During Hemodialysis .What can we Do? New Approachs for Treatment and Preventing. Eur J Gen Med 2015; 12(3):277-281

[7]. Judith Z. ( 2012). Review of Hemodialysis for Nurses and Dialysis Personnel. Elsevier Mosby,USA, 8th , edition.

[8]. Kouidi E.J, Grekas D.M, Deligiannis A.P.(2009). Effects of exercise training on noninvasive cardiac measures in patients undergoing long-term hemodialysis: a randomized controlled trial. American Journal of Kidney Diseases;54:511-21.

[9]. Khosroshahi, et al., 2013. Comparison of Vitamin E and L-Carnitine, Separately or in Combination in Patients with Intradialytic Complications. Nephro-Urology Monthly. 2013 September; 5(4):862-5.

[10]. Cheema BS, Smith BC, Singh MA.(2005). A rationale for intradialytic exercise training as standard clinical practice in ESRD. American Journal of Kidney Diseases ;45:912-6

[11]. Segura-Ortí E.(2010). Exercise in haemodyalisis patients: a literature systematic review. Nefrologia ;30:236-46.

[12]. Mahrova , A. \& Svagrove , K.(2013). Exercise Exercise Therapy - Additional Tool for Managing Physical and Psychological Problems on Hemodialysis. open access article distributed under the terms of the Creative Commons Attribution License (http://creativecommons.org/licenses/by/3.0), which permits unrestricted use, distribution, and reproduction in any medium, provided the original work is properly cited.pp.810-815

[13]. Daul A.E, Schäfers RF, Daul K, Philipp T.(2004). Exercise during hemodialysis. Clinical Nephrology ;61(Suppl 1):S26-30.

[14]. Avesani C-M, Trolonge S, Del' eavak P.(2012) Physical activity and energy expendi- ture in haemodialysis patients: aninternational survey. Nephrol Dial Transplant; 27: 2430-2434.

[15]. Cordeiro, A, C, Carrero , J.J , Queshi, A ,R, Cunha , R. F, Lindholm ,B.,Castro , I. D,\& Noronha , I. L. (2013). Study of the incidence of dialysis in Sao Paulo, the largest Brazilian city. Clinics. 68(6):760-765.

[16]. Ahmed, A, Allam , M., Habil, E , Metwally , A., Ibrahim ,N.,Radwan , M. \& Gadallah , M.(2010). Development of practice quidlines for hemodialysis in Eygpt. India Jounal of nephrology . 20(4):194.

[17]. Nieswiadomy, R. (2012). Foundations of Nursing Research. (6th ed.). Person prentice hall. Upper Saddle River .pp:114-1129.

[18]. Intra-dialysis stretching leg exercises for cramps.(2013). Research exercise therapy center, faculty of physical education. Charles University. Prague.

[19]. Mastnardo,D. Lewis,J., Hall K., Sullivan C., Cain K., Theurer J., Huml A. and Sehgal A.,(2016). Intradialytic Massage for Leg Cramps Among Hemodialysis Patients: a Pilot Randomized Controlled Trial. International Journal of therapeutic Massage and Bodywork-Volume 9, number 2, June 2016

[20]. Oh-Park M, Fast A, Gopal S, Lynn R, Frei G, Drenth R, et al.(2002). Exercise for the dialyzed: aerobic and strength training during hemodialysis. Am Journal of Physical Medicine rehabilitation ;81:814-21

[21]. Chen JL, Godfrey S, Ng TT, Moorthi R, Liangos O, Ruthazer R, et al.(2010). Effect of intra-dialytic, low-intensity strength training on functional capacity in adult haemodialysis patients: a random-ized pilot trial. Nephrol Dial Transplant;25:1936-43.

[22]. Isaac S. and Jacob D.(2016). Effectiveness of intradialytic stretching exercise on muscle cramps among patients undergoing hemodialysis. Asian Journal of Phytomedicine and Clinical Research. 4(2), 2016, 80 - $86 . \quad$ Available online: www.uptodateresearchpublication.com

[23]. Macdonald JH, Marcora SM, Jibani M, Phanish MK, Holly J, Lemmey AB.(2005). Intradialytic exercise as anabolic therapy in haemo-dialysis patients a pilot study. Clinical Physical Function Imaging ;25:113-8.

[24]. Mohamed M., Ahmed A. and Abo Zead S. (2007). impact of stretching exercises protocol on reduction of muscle cramping during haemdialysis, among chronic renal failure patients'. AMJ, Vol. 5, N. 2, April, 2007

[25]. Gowthami (2012). The effectiveness of intradialytic stretching exercise on muscle cramps among patients undergoing hemodialysis", Journal of TNNMUV, 20(1), 40-43.

[26]. Sullivan C. (2013). Investigating the effectiveness of intradialytic massage on cramping in dialysis patients, Journal of Metro health medical centre, 5(1), 2013, 4546 